\title{
Lipoprotein cholesterols are stored in high-resistant, metastatic cancer cells and released upon stress: implication for a mechanism underlying hypocholesterolemia in cancer patients
}

Taka Eguchi1,2,3,*, Chiharu Sogawa ${ }^{1,3}$, Kisho Ono $^{1}$, Mami Itagaki1,3, Masaki Matsumoto $^{1}$

1Department of Dental Pharmacology, Graduate School of Medicine, Dentistry and Pharmaceutical Sciences, Okayama University, Okayama, Japan.

${ }^{2}$ Advanced Research Center for Oral and Craniofacial Sciences, Graduate School of Medicine, Dentistry and Pharmaceutical Sciences, Okayama University, Okayama, Japan.

30kayama University Dental School, Okayama, Japan.

${ }^{4}$ Department of Molecular and Cellular Biology, Medical Institute of Bioregulation, Kyushu University, 3-1-1 Maidashi, Higashi-ku, Fukuoka 812-8582, Japan.

${ }^{*}$ Correspondence and requests for materials should be addressed to:

Taka Eguchi, D.D.S., Ph.D.

2-5-1 Shikata-cho, Okayama 700-8525 Japan

Phone: +81-86-235-6662; Fax: +81-86-235-6664

E-mail: eguchi@okayama-u.ac.jp

Author Contributions: TE conceptualized and designed the study. TE, CS, and MM prepared resources. TE, MM, CS, KO devised methodology. CS, MM, KO, MI carried out the experimentation. TE, KO, CS, MM interpreted data. TE wrote the manuscript. KO, CS revised and edited the manuscript. All authors reviewed the manuscript. 


\begin{abstract}
Resistant cancer often shows a particular secretory trait such as heat shock proteins (HSPs) and extracellular vesicles (EVs), including exosomes and oncosomes surrounded by lipid bilayers. Lipoproteins are biochemical assemblies that transport hydrophobic lipid (a.k.a. fat) molecules in body fluid and are composed of a single-layer phospholipid and cholesterol outer shell, lipids molecules within the particles, and apolipoproteins embedded in the membrane. However, lipoprotein storage and secretion by cancer cells have not well-investigated yet. We found lipoproteins were stored and abundantly secreted by neuroendocrine, castration-resistant prostate cancer (NEPC / CRPC) cells but barely secreted by colon cancer cells and oral squamous cell carcinoma (OSCC) cells. In addition, large EVs (approx. $300 \mathrm{~nm}$ diameter) and potential oncosomes were released by CRPC and OSCC cells. Proteomics revealed that CRPC cells secreted EVs enriched with tetraspanins and extracellular matrices which were reduced upon heat shock stress and alternatively lipoproteins and HSPs were secreted upon stress. Heat shock stress triggered secretion of lipoprotein-EV complexes that contained apolipoprotein A, B, C and E. These data suggested that vesicular assembly composed of EVs and lipoproteins enriched with cholesterols and phospholipids may be stored in resistant cancer cells but released upon cell stress that is increased in cancer therapies.
\end{abstract}

Keywords: Lipoprotein, extracellular vesicles, exosome, ectosome, stress response, resistant cancer, metastatic cancer, heat shock stress.

Abbreviations: CRPC, castration-resistant prostate cancer; EV, extracellular vesicle; HDL, highdensity lipoprotein; HSP, heat shock protein; LDL, low-density lipoprotein; NEPC, neuroendocrine prostate cancer; OSCC, oral squamous cell carcinoma; VLDL, very low-density lipoprotein. 


\section{INTRODUCTION}

Resistant cancer cells often show particular secretory phenotypes such as heat shock proteins (HSPs) and extracellular vesicles (EVs), including exosomes [Eguchi et al., 2018b; Fujiwara et al., 2018a; Ono et al., 2018]. HSPs play cytoprotective roles by folding unfolded and misfolded proteins, which protect normal cells against apoptosis, although cancer cells can be also protected from cytotoxic therapies and immune system [Eguchi et al., 2017; Eguchi et al., 2018a; Murshid et al., 2013]. Indeed, elevated levels of HSPs have been found in many types of cancer [Ciocca and Calderwood, 2005; Ciocca et al., 1993; Ciocca et al., 2013]. We showed that castration-resistant, neuroendocrine prostate cancer (CRPC / NEPC) cells secrete HSP90 $\alpha$ when formed grape-like multi-cellular aggregation with cancer-initiating cell (CIC) traits [Eguchi et al., 2018b]. We also showed elevated levels of HSP90 $\alpha$ and HSP90 $\beta$ in EVs secreted by metastatic oral squamous cell carcinoma (OSCC) cells compared with the low-metastatic parental OSCC cells [Ono et al., 2018]. Therefore, secretion of HSPs can be a particular secretory phenotype of resistant cancer cells. It has been shown that heat shock transcription factor 1 (HSF1) upregulates HSPs in cancer and in response to cell stress [Chou et al., 2015; Eguchi et al., 2017]. Activating phosphorylation of HSF1 at Serine 326 residue by mTOR signaling was found in breast cancer and targeting of HSF1 and mTOR by siRNA reduced levels of $\beta$-catenin, IncRNA-p21, and mammosphere formation, which is a trait of CICs [Chou et al., 2015]. Thus, increased stress signaling can be crucial in resistant cancer. Cancer therapies can trigger therapeutic stress signaling as well. We showed that anti-EGFR therapeutic antibody cetuximab was secreted with EVs by OSCC cells, which might be a therapeutic stress resistant secretory response [Fujiwara et al., 2018a].

Lipoproteins can be also involved in stress response and resistance in cancer. Lipoproteins are biochemical assemblies that transport hydrophobic lipid (a.k.a. fat) molecules in body fluid and are composed of a "single-layer" phospholipid and cholesterol outer shell, lipids molecules within the particles, and apolipoproteins embedded in the membrane [Thaxton et al., 2016]. Cholesterols are known to be released from cells as forms of lipoproteins. Types of lipoproteins and their approximate size are as follows: HDL (10 nm), LDL (20 nm), VLDL (30 to $200 \mathrm{~nm}$ ), and chylomicrons (70 $\mathrm{nm}$ to $1 \mu \mathrm{m}$ ) (Table S1), whereas EVs such as exosomes (50 to $200 \mathrm{~nm}$ ), shed microvesicles (MV, a.k.a. ectosomes, 100 to $500 \mathrm{~nm}$ ), apoptotic bodies ( 0.8 to $5 \mu \mathrm{m}$ ), and large oncosomes ( 1 to $10 \mu \mathrm{m}$ ) were surrounded by "lipid bilayered membranes" [Minciacchi et al., 
2017] (Table S2). EVs were also classified according to their sizes as small EVs and large EVs [Durcin et al., 2017]. We recently showed that depletion of lipid cholesterol efflux pump ABCG1 triggered the intracellular accumulation of extracellular vesicles and reduces tumorigenesis of metastatic adenocarcinoma cells [Namba et al., 2018]. It has been shown that obesity is a risk of cancer incidence [Suzuki et al., 2013; Tanaka et al., 2012; Yoshimoto et al., 2013] while hypocholesterolemia is a prognostic marker of gastric cancer, colorectal cancer (CRC), and oral cancer [Cengiz et al., 2006; Chawda et al., 2011; Tamura et al., 2012].

In the present study, we found that lipoproteins could be profoundly secreted by CRPC cells and metastatic adenocarcinoma cells but barely by OSCC cells, suggesting cancer type-specific lipoprotein storage and secretory traits. Therefore, we aimed to study whether high-resistant, metastatic cancer cells are able to store lipoproteins and secrete these in response to cell stress. Storage of lipoproteins in cancer cells can be a cause of hypocholesterolemia in cancer patients and stress-responsive secretion of lipoproteins and EVs can be involved in resistant traits of cancer.

\section{RESULTS}

\section{Apolipoproteins were secreted by CRPC / NEPC cells.}

We have shown cancer cells secreted molecular chaperones and exosomes that might contain particular secretory traits. We in the present study examined deep proteomics of EVs secreted by PC-3 CRPC cells. The EV fraction of CRPC cells contained profound levels of apolipoproteins, HSPs, and their receptor CD91/LRP1 (Fig 1A). Their MS scores and rank were ApoB (rank \#11, score 1574.6), HSP90 $\alpha$ (rank \#35, score 670.0), CD91/LRP1 (rank \#42, score 670.0), HSP70.1 (rank \#103, score 306.5), ApoE (rank \#122, score 252.2), ApoCIII (rank \#127, score 245.6), EpCAM (rank \#133, score 236.1), tetraspanin 1 / CD63 (rank >200, score 87.8), and ApoCII (rank $>200$, score 37.1) (Fig 1B). Therefore, it was suggested that apolipoproteins were secreted by CRPC cells with lipoprotein particles that can be associated with EVs.

\section{Detection of extracellular vesicles and lipoproteins.}

Several methods for EV preparation have been established as follows: ultra-centrifugation (UC) method, polymer-based precipitation (PBP) [Fujiwara et al., 2018a; Ono et al., 2018], affinity capturing [Nakai et al., 2016], sucrose density gradient, size exclusion chromatography, 
filtration, immunological separation, isolation by sieving, and their combinations [Lobb et al., 2015; Lotvall et al., 2014; Witwer et al., 2013]. These methods have distinct mechanisms of EV preparation with advantages and disadvantages. We have tested PBP and UC methods for isolation of EVs [Ono et al., 2018] and have established a modified PBP method combined with efficient filtration (Fig 2 a, b). We in the present study used the modified PBP method using 0.2$\mu \mathrm{m}$ pore-filter (Fig 2a) and the modified PBP method without $0.2-\mu \mathrm{m}$ pore-filter (Fig 2b). Differential sized EV fractions were prepared by using the method "a" and " $b$ " from the conditioned media of OSCC cells (Fig 2c, top two graphs). An EV fraction with a single peak at $160 \mathrm{~nm}$ diameter were prepared using the method "a" whereas another EV fraction with two peaks at $140 \mathrm{~nm}$ and at $1900 \mathrm{~nm}$ diameters were prepared using the method "b", suggesting that large EVs and/or oncosomes were secreted by OSCC cells and the $0.2-\mu \mathrm{m}$-filtration could remove the large EVs/oncosomes.

EV secretory phenotypes were different among OSCC cells, CRC cells, and CRPC cells. Size of EVs derived from CRC Colon26 cells using the method "b" was peaked at $150 \mathrm{~nm}$ with a gentle slope reaching $20 \mathrm{~nm}$ diameter (Fig 2c, third graph), indicating that this fraction could contain small EVs (exosomes), MVs, and LDL. Size of EVs derived from CRPC PC-3 cells using the method "b" were peaked at $6 \mathrm{~nm}, 60 \mathrm{~nm}$, and $390 \mathrm{~nm}$ (Fig 2c, bottom), indicating that CRPC cells secreted small EVs (approx. $60 \mathrm{~nm}$ ), large EVs (approx. 300 to $400 \mathrm{~nm}$ ), and HDLs (5 to $10 \mathrm{~nm}$ ). The particles sized at approx. $390 \mathrm{~nm}$ could be potentially large EVs or small EVs associated with many lipoproteins (see later TEM and SEM images).

To confirm EVs visually, we used transmission electron microscopy (TEM) and scanning electron microscopy (SEM). EVs secreted by Colon26 cells appeared to be 100 -nm cup-like shapes surrounded by a thin membranes or thick membranes (Fig 3a, left panel). (An EV on the left was appeared to be surrounded by a thin membrane. Another EV on the right was appeared to be surrounded by a thicker membrane.) An EV fraction prepared from conditioned media of PC-3 CRPC cells contained cup-like structured EVs but also abundantly HDL-like 10-nm particles (Fig 3a, center and right), consistent with the data of particle diameter distribution analysis shown in Fig 2c. Large EVs sized approx. $300 \mathrm{~nm}$ diameter were also found in the EV fractions derived from OSCC HSC-3 cells and CRPC PC-3 cells (Fig 3b). The 300-nm EVs derived from HSC3 OSCC cells were appeared to be surrounded by multi-layered membranes (Fig 3b, left). 
Lipoprotein-like particles were found inside the 300-nm large EVs (Fig 3b). Association of the 300-nm large EVs with small EVs were found under TEM (Fig 3b, arrows).

These data indicated that CRPC cells, OSCC cells, CRC cells secrete exosomes and large EVs (approx. $300 \mathrm{~nm}$ ) but also secrete lipoproteins and oncosomes.

\section{Cell stress triggered the increase in lipoprotein-bound EVs secreted by CRPC cells.}

It was shown that cholesterols could be stored in prostate cancer cells and other cancer cells. Cholesterols are known to be released from cells as forms of lipoproteins. We hypothesized that stored cholesterols could be released from CRPC cells as forms of lipoproteins, which is composed of cholesterols, apolipoproteins, phospholipid, and triglyceride. We next counted the number of such small particles associated with EVs secreted by CRPC cells with or without heat shock stress (HS). The number of small particle-associated EVs were increased in response to HS of PC-3 CRPC cells (Fig 3 c, d). We hypothesized that components of lipoproteins such as cholesterols and phospholipid could be supplied by the serum that was used in pre-culture of the CRPC cells, which could store these components, although we prepared EVs from the serumfree medium. We therefore next compared the number of small particles associated with EVs secreted by CRPC cells that were pre-cultured with $2 \%$ or $10 \%$ serum. The number of such EVassociated particles were larger in the $10 \%$-serum pre-cultured condition compared with the 2\%-serum pre-cultured condition (Fig 3c, left two bars).

We next compared such EV-associated particles among different types of cancer cells: CRPC PC3 cells [Eguchi et al., 2018b], OSCC HSC-3 cells [Fujiwara et al., 2018a; Ono et al., 2018], CRC Colon26 cells, and its high-metastatic subline LuM1 cells [Namba et al., 2018; Okusha et al., 2018]. The average number of EV-associated particles was 4.7 particles (CRPC PC-3 cells), 0.4 particles (OSCC HSC-3 cells), 1.3 particles (CRC Colon26 cells), and 4.0 particles (high-metastatic CRC LuM1 cells) (Fig 3c), suggesting that cholesterol secretion and association to EVs were associated with cancer types. It was worthy of note that PC-3 and LuM1 were more metastatic than HSC-3 or Colon26, respectively. Coincidently, more EV-lipoprotein complex was secreted by these high-metastatic cells.

These findings indicated that lipoprotein-bound EVs were secreted by high-metastatic cells and secreted in response to cell stress. 


\section{Apolipoproteins were secreted by the CRPC cells in response to cell stress.}

We next examined proteome traits of CRPC-EVs and their alteration in stress response. EV protein concentration ( $\mu$ g per a million cells) was increased in response to HS (Fig 4a), suggesting that EVs were released by CRPC cells in stress response. Consistently, cellular protein concentration ( $\mu \mathrm{g}$ per a million cells) was also increased in stress response (Fig 4b), suggesting that protein synthesis and/or EV uptake could be increased in stress response. Levels of EpCAM and CD9 in EVs were increased upon HS (Fig 4 c, d), suggesting that exosomes were secreted in response to HS. We next visualized proteins separated in polyacrylamide gel. Significant increases in some protein species in response to cell stress were found (Fig 4e). We therefore next examined alteration of proteome signatures of the CRPC-EVs in response to HS. We classified proteins by their types. The levels of metabolic enzymes, tetraspanins, extracellular matrix, extracellular signaling molecules, and cytoskeletal proteins were high in the CRPC-EVs without HS, although levels of these types of proteins were decreased in stress response (Fig 4f, Table 1, 2). Conversely, levels of membrane-associated proteins, molecular chaperones, apolipoproteins, and poly-ubiquitin were increased in CRPC-EVs with stress response. We next investigated types of apolipoproteins, main components of lipoproteins, found in the CRPC-EVs. Apolipoprotein A, B, and E (ApoA, ApoB, and ApoE) were found in the CRPC-EVs and their levels were increased in response to cell stress (Fig 4g). Thus, it was indicated that secretion of EV, lipoproteins, and EV-lipoprotein complex was triggered by cell stress of CRPC cells.

\section{Discussion}

We showed that apolipoproteins were abundantly secreted with EVs by CRPC cells (Fig 1-4). Lipoprotein-like single-layered particles with 5 to $30 \mathrm{~nm}$ were associated with EVs derived from the CRPC cells (Fig 3), indicating a particular lipoprotein secretory phenotype of high-resistant, metastatic cancer. Apolipoproteins-EV complex was also secreted by high-metastatic CRC cells but barely by OSCC HSC-3 cells (Fig 2, 3). HS increased secretion of EVs, lipoproteins and their assembled complex by CRPC cells (Fig 3). These data indicated that high-resistant, highmetastatic cancer cells stores lipoproteins but release them with EVs upon cell stress (Fig 5). It has been known that cancer patients were often accompanied with hypocholesterolemia [Chawda et al., 2011]. It was shown that uptake of LDL was elevated in drug-resistant human leukemic cell lines [Tatidis et al., 2002]. These reports fit our results that resistant cancer cells 
stored cholesterols which are the main components of lipoproteins. We recently showed that OSCC cells are able to secrete molecularly targeting antibody medications with exosomes, which could drive medication resistance [Fujiwara et al., 2018a]. This exosome-based resistant mechanism of cancer may also escape from cancer immunotherapy. However, anti-EGFR antibody cetuximab could capture EGFR-contained exosomes and then inhibit carcinogenic epithelial-mesenchymal transition (EMT), indicating a novel mechanism of action underlying molecularly targeting therapeutics that capture oncogenic proteins represented on the surface of exosomes [Fujiwara et al., 2018b]. However, lipoproteins bound on the surface of exosomes are able to mask such oncoproteins, indicated by the present study. We therefore are investigating further roles of lipoproteins that could associate with exosomes and other EVs.

In cancer clinical cases, blood HDL-cholesterol (HDL-C) and LDL-cholesterols (LDL-C) levels are often lowered after malignancy. Low levels of blood HDL-C and LDL-C are prognostic biomarkers of acute lymphoblastic leukemia [Baroni et al., 1996]. Hypocholesterolemia is a prognostic marker of gastric cancer and colon cancer [Cengiz et al., 2006; Tamura et al., 2012]. Increased cholesterol uptake is required for tumor cell growth and maintenance mediated by activation of LDL-R [Ho et al., 1978]. In our study, CRCP cells stored lipid and lipoproteins and secreted in the forms of EVs and lipoproteins. This manner of CRCP cells may be similar to clinical tumors. Our studies also touch upon cell stress-induced secretion of EV-lipoprotein complex. ApoA, ApoB, and ApoE were found to be secreted from the CRCP cells. Such a range of apolipoprotein indicates CRPC secretion of a range of lipoproteins, including HDL, LDL, VLDL, and chylomicron. Therefore, it was indicated that CRCP cells store a large range of lipoproteins. The lipoproteins were secreted upon cell stress with EVs associated with 5 to 20 lipoprotein particles. This EV-lipoprotein interaction may be involved in hypocholesterolemia found in cancer. Retroactively, hypercholesterolemia is a risk factor for the onset of cancer. It has been shown that statin, an anti-hypercholesterolemia drug, reduces blood cholesterol via inhibition of liver HMG-CoA reductase [Quesney-Huneeus et al., 1979]. Stain also inhibited cancer, including prostate cancer, pancreatic cancer, ovarian cancer, and colon cancer. Cancer cholesterol metabolism and secretion may be inhibited by the stain. Yet, we are currently investigating further mechanism and significance underlying EV-cholesterol crosstalk.

Our studies also indicate switching of the secretory phenotype of resistant cancer upon HS. HS switched the secretory phenotype from tetraspanin-rich, ECM-rich trait to the chaperone-rich, 
lipoprotein-rich trait (Fig 4). We recently showed that OSCC-EVs were enriched with ECM which were lost in metastatic OSCC-derived EVs in which instead chaperones were enriched [Ono et al., 2018]. Thus, metastatic traits may be associated with increased stress signals such as HSF1HSPs axis. Metastatic cancer cells robustly produce matrix metalloproteinases that can degrade ECM around the cells as well as EVs [Eguchi et al., 2008; Okusha et al., 2018; Ono et al., 2018]. We are currently investigating whether stress signal can activate MMPs that arrange intracellular and extracellular milieu.

In conclusion, our data suggested that vesicular assembly composed of EVs and lipoproteins enriched with cholesterols and phospholipids could be stored in high-resistant, high-metastatic cancer cells, which could cause hypocholesterolemia in cancer patients, but a secretory phenotype of lipoprotein-EV complex by such malignant cancer types could be increased in response to cell stress that is often increased in cancer therapies.

\section{MATERIALS AND METHODS}

Cell culture and heat shock stress. CRPC /NEPC cell line PC-3, CRC cell line Colon26, and its high-metastatic subline LuM1 were maintained in RPMI1640 medium with 10\% FBS [Eguchi et al., 2018b; Okusha et al., 2018]. OSCC cell line HSC-3 was maintained in DMEM with 10\% FBS [Fujiwara et al., 2018a; Ono et al., 2018]. For HS, cells were incubated at $43^{\circ} \mathrm{C}$ for 1.5 or 3 hours as described [Clayton et al., 2005; Eguchi et al., 2017] (Fig S1).

Preparation and analysis of EVs. EV fractions were prepared using a modified polymerprecipitation method as described [Eguchi et al., 2018b; Ono et al., 2018]. Transmission electron microscopy (TEM), particle diameter distribution analysis, and protein assay were carried out as described [Ono et al., 2018]. Numbers of lipoprotein-like particles in the TEM images were counted. For scanning electron microscopy (SEM), $5 \mu \mathrm{lEV}$ fraction was dropped on a cover glass and dried at $40^{\circ} \mathrm{C}$ for $20 \mathrm{~min}$. The EV samples and cover glass were coated with Osmium. SEM images were taken using Hitachi S-4800 at Okayama University Medical School Central Research Laboratory.

\section{Identification of proteins in exosome fraction by LC-MS/MS.}

Exosome fraction was incubated in the presence of $1 \%$ SDS and $2.5 \mathrm{mM}$ Tris (2- 
carboxyethyl)phosphine hydrochloride (Thermo Fisher Scientific) for $10 \mathrm{~min}$ at $85^{\circ} \mathrm{C}$ followed by alkylation with $12.5 \mathrm{mM}$ iodoacetamide (Sigma) for $15 \mathrm{~min}$ at room temperature. Proteins were precipitated with acetone for $2 \mathrm{~h}$ at $-30^{\circ} \mathrm{C}$ and resulting pellet was dispersed in $100 \mathrm{mM}$ ammonium bicarbonate by ultrasonic treatment (three times for $30 \mathrm{~s}$ with intervals of $30 \mathrm{~s}$ ) with a Bioruptor (Diagenode). The protein suspension was subjected to digestion with trypsin (1 $\mu \mathrm{g}$, Wako) for $14 \mathrm{~h}$ at $37^{\circ} \mathrm{C}$.

Resulting peptides were analyzed by QExactive mass spectrometer that was coupled with nanoLC (AdvanceLC, Michrom Inc.) via a nano-electrospray source with a column oven set at $37^{\circ} \mathrm{C}$ (AMR Inc.). Samples were injected to pre-column (L-column micro: $0.3 \mathrm{~mm}$ inner diameter, 5 mm length, CERI Japan) and separated by in-house made $20 \mathrm{~cm}$ column (inner diameter $100 \mu \mathrm{m}$, $3 \mu$ L-column, CERI, Japan) with a linear gradient (5-30\% B for $110 \mathrm{~min}, 30-90 \%$ B for $1 \mathrm{~min}$, and $90 \%$ B for $10 \mathrm{~min}$, A: $0.1 \%$ formic acid, $2 \%$ acetonitrile, B: $0.1 \%$ formic acid, $99.9 \%$ acetonitrile) at a flow rate of $250 \mathrm{nl} / \mathrm{min}$. The QExactive was operated in data-dependent acquisition mode. Scan ranges were set at m/z 375-1600 for MS spectra and at m/z 200-2000 for MS/MS spectra, respectively. MS spectra were acquired at a resolution of 70,000 at $\mathrm{m} / \mathrm{z} 400$ after accumulation to a target value of $1 \times 10^{6}$ with the maximum ion injection times for $60 \mathrm{msec}$. Up to the top 10, most abundant ions with charge 2+ or 3+ from the survey scan were selected with an isolation window of $1.5 \mathrm{~h}$ and fragmented by HCD with normalized collision energies of 25. MS/MS spectra were acquired at a resolution of 17,500 at m/z 400 after accumulation to a target value of $5 \times 10^{4}$ with the maximum ion injection times for $120 \mathrm{msec}$. The acquired MS/MS spectra were analyzed by Proteome Discoverer 1.4 with the Mascot algorithm (ver. 2.6.0) using an IPI human database (ver. 3.8.7). The search was performed with the following parameters: Trypsin was selected as an enzyme used, allowed a number of missed cleavages was set 3 and carbamidomethylation on Cys were selected as a fixed modification. Oxidized methionine was searched as variable modifications. Precursor mass tolerances were $10 \mathrm{ppm}$ and tolerance of MS/MS ions was 0.02 Da. The second LC-MS/MS with or without HS was carried out as described [Eguchi et al., 2008; Ono et al., 2018].

SDS-PAGE and in-gel protein staining. EV protein samples were mixed with the SDS sample buffer containing $\beta$-mercaptoethanol and then heated at $95{ }^{\circ} \mathrm{C}$ for $5 \mathrm{~min}$. Protein samples were separated by SDS-PAGE in 4-20\% TGX-GEL (BioRad). The gel was stained with 
Oriole fluorescent gel stain solution (BioRad) for $90 \mathrm{~min}$.

\section{Acknowledgements}

The authors thank Yuka Okusha and Kuniaki Okamoto for discussion and technical assistance.

Funding: This work was supported by JSPS KAKENHI, grant numbers JP17K11642 (to TE), 17K11643 (to CS TE), JP16K11722 (toTE), and JP17K11669 (to TE).

Competing Interests: The authors have no competing financial interests to declare.

Table 1. HS-induced secretion with EVs.

\begin{tabular}{lrr} 
Protein name & Alias & \multicolumn{2}{c}{ Secretion } \\
\hline Apolipoprotein A1 & ApoA1 & 24.1 \\
Heat shock protein 90 & HSP90 & 16.7 \\
Apolipoprotein E & ApoE & 15.5 \\
Prothrombin & PT & 13.4 \\
Transmembrane protein 44 & TMEM44 & 13.3 \\
Wingless-type MMTV integration site 2B & WNT-2B & 13.1 \\
Ankyrin repeat domain 31 & ANK31 & 13.0 \\
Annexin A2 & AXNA2 & 11.3 \\
Apolipoprotein B & ApoB & 6.1 \\
Poly Ubiquitin & Poly Ub & 2.8
\end{tabular}

Table 2. HS-dependent reduction within EVs.

\begin{tabular}{lrr} 
Protein name & Alias & \multicolumn{2}{c}{ HS-dependent } \\
\hline Actin & ACTN & 53.4 \\
$\alpha$-2-heparan sulfate-glycoprotein & AHSG & 23.3 \\
Tetraspanin-28 / Cluster of differentiation 81 & CD81/TSPAN28 & 18.7
\end{tabular}


Tenascin C

TNC

Phosphodiesterase 4C

PDE4C

14.1

Procollagen-lysin,2-oxoglutarate 5-dioxygenase 1

PLOD1

a2-Macroglobulin

A2M

12.1

Fibronectin

FN

Keratin 10

KRT10

Thrombospondin 1

TSP1

2.0

\section{FIGURE LEGENDS}

Fig 1. High-sensitive proteomics of EVs secreted by CRPC / NEPC cells. EVs were prepared from cell culture supernatant of PC-3 cells and analyzed using LC-MS/MS. (a) A rank abundance curve of protein species found in EVs. Apolipoproteins, HSPs, and LRP1/CD91 indicated in the plots. (b) A list of apolipoproteins, HSPs, LRP1, EpCAM, and tetraspanin 1/CD63 found in the PC-3-EVs.

Fig 2. EV isolation methods alter characters of EV fraction. (a, b) Flow charts of PBP methods for EV isolation with (a) or without (b) 0.2 micrometer filtration. This modified PBP method was established previously [Ono et al., 2018]. (c) Particle diameter distribution analysis. EVs were prepared from conditioned media of OSCC HSC-3 cells using method "a" and method "b", colorectal cancer (CRC) Colon26 cells using method "b", and CRPC PC-3 cells using method "b".

Fig 3. Exosomes, lipoproteins, and large EVs secreted by cancer cells. (a) TEM and SEM images of EV fractions secreted by CRC Colon26 cells and CRPC PC-3 cells. Scale bars, $100 \mathrm{~nm}$. Arrows, exosomes or MVs with cup-like shapes sized at 50 to $150 \mathrm{~nm}$. Arrowheads, HDL or LDL particles sized at 5 to $30 \mathrm{~nm}$ diameter. (b) TEM images of large EVs. Scale bars, $100 \mathrm{~nm}$. The fractions were prepared from conditioned media of OSCC HSC-3 cells (left) and CRPC PC-3 with HS for 1.5 or 3 hours (center and right panels). The large EVs were sized at approx. $300 \mathrm{~nm}$. Small EVs (approx. $100 \mathrm{~nm}$ ) associating with large EVs (approx. $300 \mathrm{~nm}$ ) were indicated by arrows. (c) The number of EV-associated lipoprotein-like particles. PC-3 cells were pre-cultured in media containing 2 or $10 \%$ serum and then heat-shocked for $0,1.5$ or 3 hours (NH, HS $1.5 \mathrm{~h}$ or HS $3 \mathrm{~h}$ ). 
Media were then replaced by serum-free media. EV fractions were prepared at $24 \mathrm{~h}$ after the medium change. HSC-3, Colon26, and LuM1 cells were pre-cultured in media containing 10\% serum and then media were replaced by serum-free media. EVs were prepared from the conditioned media at 2 days after the medium change. Number of EV-associated lipoprotein-like particles in the TEM images were counted. (d) Representative TEM images of EVs associated with lipoprotein-like particles. Scale bars, $100 \mathrm{~nm}$.

Fig 4. Heat shock stress promoted secretion of apolipoproteins. PC-3 cells were heatshocked for $0,1.5$ or 3 hours and the recovered in serum-free media for 2 days. EVs were prepared from conditioned media. NH, non-heated. HS1.5, heat-shocked for $1.5 \mathrm{~h}$. HS3, heatshocked for $3 \mathrm{~h}$. (a) EV protein concentration per $1 \times 10^{6}$ cells. (b) Cellular protein concentration per $1 \times 10^{6}$ cells. (c, d) Levels of EpCAM (c) and CD9 (d) in EVs were analyzed using Exoscreen. (e) EV proteins visualized by SDS-PAGE and Oriole fluorescent gel staining. (f) Alteration of EV proteome signature in response to heat shock stress. EV proteins identified by LC-MS/MS were classified as described previously [Ono et al., 2018]. (g) HS-dependent inducibility of apolipoproteins identified by the proteomics.

Fig 5. Graphical abstract. Obesity is a risk factor of cancer incidence and hypercholesterolemia has been found in cancer patients. In the present study, we showed that metastatic, resistant cancer cells (PC-3 and LuM1) have abilities to store and release lipoproteins that were associated with EVs such as exosomes (left). Heat shock stress to CRPC PC-3 cells increased association of lipoproteins to EVs, which might involve secretory phenotypes of high-resistant, metastatic cancer cells in response to therapeutic stress (right).

Fig S1. Graphical protocol of heat shock and EV preparation. Cells were pre-cultured in media with $2 \%$ or $10 \%$ serum. After HS, media were replaced by serum-free media. EV fractions were prepared at day 1 or day 2 after the medium change. 
Table S1. Size of lipoproteins.

\begin{tabular}{|l|l|}
\hline Type of lipoproteins & Diameter \\
\hline HDL & $10 \mathrm{~nm}$ \\
\hline LDL & $20 \mathrm{~nm}$ \\
\hline IDL & $40 \mathrm{~nm}$ \\
\hline VLDL & $30-200 \mathrm{~nm}$ \\
\hline Chylomicrons & $70-1,000 \mathrm{~nm}$ \\
\hline
\end{tabular}

Table S2. Size of extracellular vesicles.

\begin{tabular}{|l|l|}
\hline Type of EVs & Diameter \\
\hline Exosomes & $50-200 \mathrm{~nm}$ \\
\hline Ectosomes, shed microvesicles & $100-500 \mathrm{~nm}$ \\
\hline Apoptotic bodies & $800-5,000 \mathrm{~nm}$ \\
\hline Large oncosomes & $1,000-10,000 \mathrm{~nm}$ \\
\hline
\end{tabular}

\section{References}

Baroni S, Scribano D, Zuppi C, Pagano L, Leone G, Giardina B. 1996. Prognostic relevance of lipoprotein cholesterol levels in acute lymphocytic and nonlymphocytic leukemia. Acta Haematol 96:24-8.

Cengiz 0, Kocer B, Surmeli S, Santicky MJ, Soran A. 2006. Are pretreatment serum albumin and cholesterol levels prognostic tools in patients with colorectal carcinoma? Med Sci Monit 12:Cr240-7.

Chawda JG, Jain SS, Pate1 HR, Chaduvula N, Patel K. 2011. The relationship between serum lipid levels and the risk of oral cancer. Indian J Med Paediatr Oncol 32:34-7. Chou SD, Murshid A, Eguchi T, Gong J, Calderwood SK. 2015. HSF1 regulation of betacatenin in mammary cancer cells through control of HuR/elavL1 expression. Oncogene $34: 2178-88$.

Ciocca D, Calderwood S. 2005. Heat shock proteins in cancer: diagnostic, prognostic, predictive, and treatment implications. Cell Stress \& Chaperones 10:86.

Ciocca D, Clark G, Tandon A, Fuqua S, Welch W, McGuire W. 1993. Heat shock protein hsp70 in patients with axillary lymph node-negative breast cancer: prognostic implications. J Nat1 Cancer Inst 85:570-4. 
Ciocca DR, Arrigo AP, Calderwood SK. 2013. Heat shock proteins and heat shock factor 1 in carcinogenesis and tumor development: an update. Arch Toxicol 87:19-48.

Clayton A, Turkes A, Navabi H, Mason MD, Tabi Z. 2005. Induction of heat shock proteins in B-ce11 exosomes. J Cel1 Sci 118:3631-8.

Durcin M, Fleury A, Taillebois E, Hilairet G, Krupova Z, Henry C, Truchet S, Trotzmuller M, Kofeler H, Mabilleau G, Hue 0, Andriantsitohaina R, Martin P, Le Lay S. 2017. Characterisation of adipocyte-derived extracellular vesicle subtypes identifies distinct protein and lipid signatures for large and small extracellular vesicles. J Extrace11 Vesicles 6:1305677.

Eguchi T, Calderwood SK, Takigawa M, Kubota S, Kozaki KI. 2017. Intracellular MMP3 Promotes HSP Gene Expression in Collaboration With Chromobox Proteins. J Cell Biochem 118:43-51.

Eguchi T, Kubota S, Kawata K, Mukudai Y, Uehara J, Ohgawara T, Ibaragi S, Sasaki A, Kuboki T, Takigawa M. 2008. Novel transcription-factor-like function of human matrix metalloproteinase 3 regulating the CTGF/CCN2 gene. Mol Cell Biol 28:2391-413.

Eguchi T, Lang BJ, Murshid A, Prince T, Gong J, Calderwood SK. 2018a. Regulatory roles for Hsp70 in cancer incidence and tumor progression. In Galigniana MD, editor editors. Frontiers in Structural Biology. Bentham Science, p 1-22.

Eguchi T, Sogawa C, Okusha Y, Uchibe K, Iinuma R, Ono K, Nakano K, Murakami J, Itoh M, Arai K, Fujiwara T, Namba Y, Murata Y, Shimomura M, Okamura H, Takigawa M, Nakatsura T, Kozaki K, Okamoto K, Calderwood S. 2018b. Organoids with Cancer Stem Ce11-1ike Properties Secrete Exosomes and HSP90 in a 3D NanoEnvironment. PLOS ONE 13:e0191109.

Fujiwara T, Eguchi T, Sogawa C, Ono K, Murakami J, Ibaragi S, Asaumi J, Okamoto K, Calderwood S, Kozaki K. 2018a. Anti-EGFR antibody cetuximab is secreted by oral squamous cell carcinoma and alters EGF-driven mesenchymal transition. Biochem Biophys Res Commun. :pii: S0006-291X (18) 31530-4.

Fujiwara T, Eguchi T, Sogawa C, Ono K, Murakami J, Ibaragi S, Asaumi J-i, Calderwood SK, Okamoto K, Kozaki K-i. 2018b. Carcinogenic epithelial-mesenchymal transition initiated by oral cancer exosomes is inhibited by anti-EGFR antibody cetuximab. Oral Oncology 86:251-257.

Ho YK, Smith RG, Brown MS, Goldstein JL. 1978. Low-density lipoprotein (LDL) receptor activity in human acute myelogenous leukemia cells. Blood 52:1099-114.

Lobb RJ, Becker M, Wen SW, Wong CS, Wiegmans AP, Leimgruber A, Moller A. 2015. Optimized exosome isolation protocol for cell culture supernatant and human plasma. J Extracel1 Vesicles 4:27031.

Lotvall J, Hill AF, Hochberg F, Buzas EI, Di Vizio D, Gardiner C, Gho YS, Kurochkin 
IV, Mathivanan S, Quesenberry P, Sahoo S, Tahara H, Wauben MH, Witwer KW, Thery C. 2014. Minimal experimental requirements for definition of extracellular vesicles and their functions: a position statement from the International Society for Extracellular Vesicles. J Extrace11 Vesicles 3:26913.

Minciacchi VR, Zijlstra A, Rubin MA, Di Vizio D. 2017. Extracellular vesicles for liquid biopsy in prostate cancer: where are we and where are we headed? Prostate Cancer Prostatic Dis 20:251-258.

Murshid A, Eguchi T, Calderwood SK. 2013. Stress proteins in aging and life span. Int J Hyperthermia 29:442-7.

Nakai W, Yoshida T, Diez D, Miyatake Y, Nishibu T, Imawaka N, Naruse K, Sadamura Y, Hanayama R. 2016. A novel affinity-based method for the isolation of highly purified extracellular vesicles. Sci Rep 6:33935.

Namba Y, Sogawa C, Okusha Y, al e. 2018. Depletion of lipid efflux pump ABCG1 triggers the intracellular accumulation of extracellular vesicles and reduces aggregation and tumorigenesis of metastatic cancer cells. Frontiers in Oncology 8.

Okusha Y, Eguchi T, Sogawa C, Okui T, Nakano K, Okamoto K, Kozaki KI. 2018. The intranuclear PEX domain of MMP involves proliferation, migration, and metastasis of aggressive adenocarcinoma cells. J Cell Biochem.

Ono K, Eguchi T, Sogawa C, Calderwood SK, Futagawa J, Kasai T, Seno M, Okamoto K, Sasaki A, Kozaki KI. 2018. HSP-enriched properties of extracellular vesicles involve survival of metastatic oral cancer cells. J Cell Biochem.

Quesney-Huneeus V, Wiley MH, Siperstein MD. 1979. Essential role for mevalonate synthesis in DNA replication. Proc Natl Acad Sci U S A 76:5056-60.

Suzuki S, Kojima M, Tokudome S, Mori M, Sakauchi F, Wakai K, Fujino Y, Lin Y, Kikuchi S, Tamakoshi K, Tamakoshi A. 2013. Obesity/Weight Gain and Breast Cancer Risk: Findings From the Japan Collaborative Cohort Study for the Evaluation of Cancer Risk. Journal of Epidemiology 23:139-145.

Tamura T, Inagawa S, Hisakura K, Enomoto T, Ohkohchi N. 2012. Evaluation of serum highdensity lipoprotein cholesterol levels as a prognostic factor in gastric cancer patients. J Gastroenterol Hepatol 27:1635-40.

Tanaka K, Tsuji I, Tamakoshi A, Matsuo K, Ito H, Wakai K, Nagata C, Mizoue T, Sasazuki S, Inoue M, Tsugane S, Research Group for the D, Evaluation of Cancer Prevention Strategies in J. 2012. Obesity and liver cancer risk: an evaluation based on a systematic review of epidemiologic evidence among the Japanese population. Jpn J Clin Oncol 42:212-21.

Tatidis L, Masquelier M, Vitols S. 2002. Elevated uptake of low density lipoprotein by 
drug resistant human leukemic cell 1ines. Biochem Pharmacol 63:2169-80.

Thaxton CS, Rink JS, Naha PC, Cormode DP. 2016. Lipoproteins and lipoprotein mimetics for imaging and drug delivery. Adv Drug Deliv Rev 106:116-131.

Witwer KW, Buzas EI, Bemis LT, Bora A, Lasser C, Lotvall J, Nolte-' t Hoen EN, Piper MG, Sivaraman S, Skog J, Thery C, Wauben MH, Hochberg F. 2013. Standardization of sample collection, isolation and analysis methods in extracellular vesicle research. J Extrace11 Vesicles 2.

Yoshimoto S, Loo TM, Atarashi K, Kanda H, Sato S, Oyadomari S, Iwakura Y, Oshima K, Morita H, Hattori M, Honda K, Ishikawa Y, Hara E, Ohtani N. 2013. Obesity-induced gut microbial metabolite promotes liver cancer through senescence secretome. Nature 499:97101. 